Revista Brasileira de Farmacognosia Brazilian Journal of Pharmacognosy 22(3): 490-496, May/Jun. 2012

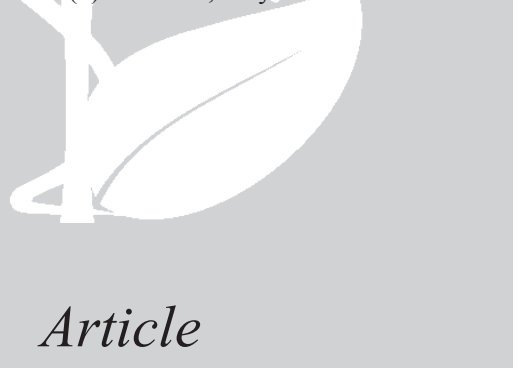

Received 21 Jun 2011

Accepted 27 Sep 2011

Available online 17 Jan 2012

Keywords:

Caragana bungei

Fabaceae

flavonoids

phenylpropanoids

$\beta$-sitosterol

ISSN 0102-695X

http://dx.doi.org/10.1590/S0102-

$695 \times 2012005000010$

\section{Chemical constituents of Caragana bungei shoots}

\author{
Daniil N. Olennikov, ${ }^{*}$, Larisa M. Tankhaeva, ${ }^{1}$ Vyacheslav $V$. \\ Partilkhaev, ${ }^{1}$ Alexander V. Rokhin ${ }^{2}$
}

${ }^{1}$ Laboratory of Medical and Biological Research, Institute of General and Experimental Biology, Siberian Division, Russian Academy of Sciences, Russian Federation,

${ }^{2}$ Department of Chemistry, Irkutsk State University, Russian Federation.

\begin{abstract}
The phytochemical study of flowering shoots of Caragana bungei Ledeb., Fabaceae, collected in Tuva Republic (Russian Federation) resulted in the isolation of sixteen compounds identified as $\beta$-sitosterol, $\beta$-sitosterol-3-Oglucoside, umbelliferone, kaempferol, quercetin, isorhamnetin-3-O-glucoside, isoquercitrin, rutin, narcissin, nicotiflorin, caffeic acid, 3- $O$-caffeoylquinic acid, 5-O-caffeoylquinic acid, 3,5-di- $O$-caffeoylquinic acid, gallic acid and sucrose. The structures of the compounds were established by spectral analyses. This is the first phytochemical investigation of $C$. bungei. The nicotiflorin, phenylpropanoids and gallic acid were isolated from the Caragana genus for the first time.
\end{abstract}

\section{Introduction}

Caragana Fabr. is a genus of Fabaceae family including about eighty species widely spread in Europe and Asia and used as a medical, decorative and technical raw materials. Despite of wide using the targeted chemical researches of plants of this genus were started relatively recently. To date we have scientific information about chemical compositions of fifteen Caragana species (Meng et al., 2009). In Russian Federation this genus presented by 34 species mainly distributed in the steppe and mountain landscapes.

Caragana bungei Lebeb. is branched spiny shrub growing in the desert steppes and valleys of mountain rivers of Eastern and Western Siberia. The decoction of flowering shoots of this species is used in traditional medicine of Tuva and Buryatia regions as an antiinflamatory and immunostimulant means (Aseeva et al., 2008). There is no any scientific data about chemical composition of $C$. bungei. In this study, we present the results of phytochemical investigation of $C$. bungei flowering shoots. As a result sixteen compounds were isolated including $\beta$-sitosterol (1), umbelliferone (2), kaempferol (3), quercetin (4), $\beta$-sitosterol-3-O-glucoside (5), caffeic acid (6), gallic acid (7), 3,5-di-O-caffeoylquinic acid (8), isorhamnetin-3-O-glucoside (9), isoquercitrin (quercetin-3-O-glucoside, 10), 3-O-caffeoylquinic acid
(11), 5-O-caffeoylquinic acid (12), rutin (quercetin-3- $O$ rutinoside, 13), narcissin (isorhamnetin-3-O-rutinoside, 14), nicotiflorin (kaempferol-3-O-rutinoside, 15) and sucrose (16). This is the first phytochemical investigation of $C$. bungei.

\section{Material and Methods}

\section{Plant material}

The shoots of Caragana bungei Ledeb., Fabaceae, were collected in flowering period in Despen locality (Tes-Hemskiľ region, Tuva Republic, Russian Federation, $\left.50^{\circ} 81^{\prime} 78^{\prime \prime} \mathrm{N}, 93^{\circ} 78^{\prime} 39^{\prime \prime} \mathrm{E}\right)$. The plant samples were identified by Prof. Aseeva T.A. (Institute of General and Experimental Biology, Ulan-Ude, Russian Federation). Voucher specimen is deposited at the Herbarium of Department of Biologically Active Substances (Institute of General and Experimental Biology, Ulan-Ude, Russian Federation; voucher specimen number $\mathrm{Fb} / \mathrm{s}-18 / 02-$ 11/0942).

\section{General experimental procedures}

Elemental composition was determined using MAT 8200 spectrometer (Thermo Finnigan). Melting 
points were determined with a 910 DS calorimeter (DuPont). UV spectra were recorded using a SF2000 spectrophotometer (OKB Specter). MS spectra were registered on a LCQ mass spectrometer (Thermo Finnigan). ${ }^{13} \mathrm{C}$ NMR spectra were recorded on a VXR 500S spectrometer (Varian). Optical rotations were measured on a DIP-1000 digital polarimeter (Jasco). Chromatography column was performed over silica gel 60 (230-400 mesh, Merck), Sephadex LH-20 (25-100 $\mu \mathrm{m}$, Pharmacia), polyamide (Woelm) and pTLC was performed on Sorbfil-A silica gel TLC plates (layer thickness $1 \mathrm{~mm}$; Imid Ltd.). All chemicals were analytical-grade. The Folin method was used to determine total phenolic content (TPC), as described by (Shetty et al., 1995) using gallic acid as a standard compound. Total flavonoid content (TFC) was determined by spectrophotometric method (Chirikova et al., 2010) using rutin as a standard compound. $\beta$-Sitosterol content was determined using HPTLC-densitometric method (Starek et al., 2007).

\section{Extraction and isolation}

Air-dried, ground shoots of C. bungei (1462 g) were extracted with $70 \% \mathrm{EtOH}$ at $80{ }^{\circ} \mathrm{C}$ three times and the extracts were concentrated under reduced pressure to yield $409.4 \mathrm{~g}$ of crude extract. The crude extract was resuspended in water $(1: 4, \mathrm{v} / \mathrm{v})$ and successively partitioned with $\mathrm{CHCl}_{3}$, EtOAc, and $n-\mathrm{BuOH}$. The organic layers were brought to dryness in vacuo to yield $87.7,43.8$ and 175.4 $\mathrm{g}$ of $\mathrm{CHCl} 3$ (CB-C), EtOAc (CB-E) and $n-\mathrm{BuOH}$ fraction (CB-B) residue respectively. The $\mathrm{CB}-\mathrm{C}$ fraction (42 g) was dissolved in $1000 \mathrm{~mL}$ of chloroform and chromatographed over Sephadex LH-20 column $(7 \times 120 \mathrm{~cm})$, eluting with $\mathrm{CHCl}_{3}$ containing increasing concentrations of $\mathrm{MeOH}$ $(100: 0 \rightarrow 0: 100)$ to obtain fifty fractions (fr.C1-fr.C50). Fr.C3-fr.C6 were combined and chromatographed on a silica column $(3 \times 50 \mathrm{~cm})$, eluting with $\mathrm{CHCl}_{3}-\mathrm{MeOH}$ mixtures $(100: 0 \rightarrow 70: 30)$ to obtain twelve fractions (fr.C36/1-fr.C3-6/12). Fr.C3-6/3 (eluent $\mathrm{CHCl}_{3}-\mathrm{MeOH}$ 90:10) after recrystallization $(\mathrm{MeOH})$ was given $263 \mathrm{mg}$ of $\mathbf{1}$. Fr.C5 was chromatographed on a silica column $(2 \times 40 \mathrm{~cm})$, eluting with $\mathrm{CHCl}_{3}-\mathrm{MeOH}$ mixtures $(90: 10 \rightarrow 60: 40)$ to obtain fifteen fractions (fr.C5/1-fr.C5/15). Fr.C5/7-fr.C5/8 were combined (eluent $\mathrm{CHCl}_{3}-\mathrm{MeOH} 74: 25$ ) and was chromatographed on a pTLC (solvent toluene-EtOAcHCOOH 6:3:1) to yield $2(15 \mathrm{mg}), 3(27 \mathrm{mg})$ and 4 (37 $\mathrm{mg})$. The CB-E fraction $(35 \mathrm{~g})$ was dissolved in $500 \mathrm{~mL}$ of $\mathrm{MeOH}$ and chromatographed over Sephadex LH-20 column $(5 \times 100 \mathrm{~cm})$, eluting with $\mathrm{MeOH}-\mathrm{H}_{2} \mathrm{O}$ mixtures $(100: 0 \rightarrow 50: 50)$ to obtain 60 fractions (fr.E1-fr.E60). Fr.E3 was chromatographed on a silica column $(2 \times 50 \mathrm{~cm})$, eluting with $\mathrm{CHCl}_{3}-\mathrm{MeOH}$ mixtures $(100: 0 \rightarrow 60: 40)$ to obtain fifteen fractions (fr.E3/1-fr.E3/15). Fr.E3/8 (eluent$\mathrm{CHCl}_{3}-\mathrm{MeOH}$ 70:30) after recrystallization (MeOH) was given $24 \mathrm{mg}$ of 5. Fr.E5-fr.E8 were combined and chromatographed on a polyamide column $(6 \times 100 \mathrm{~cm})$, eluting with $\mathrm{H}_{2} \mathrm{O}-\mathrm{MeOH}$ mixtures $(100: 0 \rightarrow 0: 100)$ to obtain twenty fractions (fr.E5-8/1-fr.E5-8/20). Fr.E5-8/2fr.E5-8/3 (eluent $\mathrm{H}_{2} \mathrm{O}-\mathrm{MeOH} 90: 10,85: 15$ ) were combined and chromatographed on a pTLC (solvent tolueneEtOAc-HCOOH 5:4:1) to yield $6(11 \mathrm{mg}), 7(8 \mathrm{mg})$ and 8 (8 mg). Fr.E5-8/4-fr.E5-8/5 (eluent $\mathrm{H}_{2} \mathrm{O}-\mathrm{MeOH}$ 80:20, $75: 25)$ were combined and chromatographed on a pTLC (solvent toluene-EtOAc-HCOOH $5: 4: 1$ ) to yield 8 (18 $\mathrm{mg})$. Fr.E11-fr.E14 were combined and chromatographed on a polyamide column $(6 \times 50 \mathrm{~cm})$, eluting with $\mathrm{H}_{2} \mathrm{O}-$ $\mathrm{MeOH}$ mixtures $(100: 0 \rightarrow 0: 100)$ to obtain twenty fractions (fr.E11-14/1-fr.E11-14/20). Fr.E11-14/11-fr.E11-14/14 (eluent $\mathrm{H}_{2} \mathrm{O}-\mathrm{MeOH}$ 60:40, 55:45) were combined and chromatographed on a pTLC (solvent EtOAc-toluene$\mathrm{HCOOH} \mathrm{6:4:1.5)} \mathrm{to} \mathrm{yield} 9(15 \mathrm{mg})$ and $\mathbf{1 0}(27 \mathrm{mg})$. Fr.E19-fr.E23 were combined and chromatographed on a polyamide column $(6 \times 100 \mathrm{~cm})$, eluting with $\mathrm{H}_{2} \mathrm{O}-\mathrm{MeOH}$ mixtures $(100: 0 \rightarrow 0: 100)$ to obtain twenty fractions (fr.E1923/1-fr.E23/20). Fr.E19-23/3-fr.E19-23/5 (eluent $\mathrm{H}_{2} \mathrm{O}-$ $\mathrm{MeOH}$ 90:10-80:20) were combined and chromatographed on a pTLC (solvent EtOAc-dioxane-AcOH- $\mathrm{H}_{2} \mathrm{O}$ 10:5:1:1) to yield $11(11 \mathrm{mg})$ and $\mathbf{1 2}(9 \mathrm{mg})$. The CB-B fraction $(87 \mathrm{~g})$ was dissolved in $1500 \mathrm{~mL}$ of $50 \% \mathrm{EtOH}$ and chromatographed polyamide column $(10 \times 120 \mathrm{~cm})$, eluting with $\mathrm{H}_{2} \mathrm{O}-\mathrm{EtOH}$ mixtures $(100: 0 \rightarrow 0: 100)$ to obtain fifty fractions (fr.B1-fr.B50). Fr.B20-fr.B23 (eluent- $\mathrm{H}_{2} \mathrm{O}-\mathrm{EtOH}$ 60:40-54:46) were combined and chromatographed on Sephadex LH-20 column $(5 \times 80 \mathrm{~cm})$, eluting with EtOH$\mathrm{H}_{2} \mathrm{O}$ mixtures $(96: 4 \rightarrow 60: 40)$ to obtain twenty fractions (fr.B20-23/1-fr.B20-23/20). Fr.B20-23/5-fr.B20-23/7 (eluent EtOH-H2O 80:20) after recrystallization (80\% EtOH) was given $86 \mathrm{mg}$ of 13. Fr.B20-23/7-fr.B20-23/9 were combined and after recrystallization $(80 \% \mathrm{EtOH})$ was given $43 \mathrm{mg}$ of 14. Fr.B27-fr.B29 (eluent $\mathrm{H}_{2} \mathrm{O}-\mathrm{EtOH}$ 46:54-42:58) were combined and chromatographed on a pTLC (solvent EtOAc-dioxane-AcOH- $\mathrm{H}_{2} \mathrm{O}$ 10:5:1:1) to yield 15 (16 mg). Fr.B1 (eluent $\mathrm{H}_{2} \mathrm{O}$ ) was concentrated to syrup which crystallized at $2{ }^{\circ} \mathrm{C}$ during 5-7 days to yield colorless prisms of $\mathbf{1 6}(53 \mathrm{mg})$.

$\beta$-Sitosterol (1): $\mathrm{C}_{29} \mathrm{H}_{50} \mathrm{O}$, m.p. $141{ }^{\circ} \mathrm{C}$. ${ }^{+} \mathrm{FAB}-\mathrm{MC} \mathrm{m} / \mathrm{z}$ : $415[\mathrm{M}+\mathrm{H}]^{+} .{ }^{13} \mathrm{C}-\mathrm{NMR}\left(\mathrm{CDCl}_{3}, 125 \mathrm{MHz}\right): 11.73(\mathrm{C}-29)$, 12.14 (C-18), 18.63 (C-26), 19.03 (C-21), 19.39 (C-19), 19.82 (C-27), 21.47 (C-11), 23.08 (C-28), 24.31 (C-15), 26.22 (C-25), 28.18 (C-16), 29.97 (C-23), 31.73 (C-2), 31.95 (C-8), 32.03 (C-7), 34.89 (C-22), 36.11 (C-20), 36.69 (C-10), 37.24 (C-1), 40.27 (C-12), 42.32 (C-4), 42.61 (C-13), 50.33 (C-24), 50.92 (C-9), 56.11 (C-17), 57.02 (C-14), 71.97 (C-3), 121.92 (C-6), 140.87 (C-5).

Umbelliferone (2): $\mathrm{C}_{9} \mathrm{H}_{6} \mathrm{O}_{3}$, m.p. $232{ }^{\circ} \mathrm{C}$. UV $\lambda_{\max } \mathrm{nm}$ MeOH: 214 sh, 248 sh, 299 sh, 327. +FAB-MC m/z: 163 $[\mathrm{M}+\mathrm{H}]+{ }^{+}{ }^{13} \mathrm{C}-\mathrm{NMR}\left(\mathrm{DMSO}-\mathrm{d}_{6}, 125 \mathrm{MHz}\right): 103.02$ (C-8), 111.60 (C-10), 111.63 (C-3), 113.27 (C-6), 129.97 (C-5), 
144.24 (C-4), 156.12 (C-9), 160.71 (C-2), 161.93 (C-7).

Kaempferol (3): $\mathrm{C}_{15} \mathrm{H}_{10} \mathrm{O}_{6}$, m.p. $275^{\circ} \mathrm{C} . \mathrm{UV} \lambda_{\max } \mathrm{nm} \mathrm{MeOH}$ : 258, $325 \mathrm{sh}, 370$. + FAB-MC m/z: $287[\mathrm{M}+\mathrm{H}]^{+} .{ }^{13} \mathrm{C}-\mathrm{NMR}$ (DMSO-d, 125 MHz): 94.38 (C-8), 99.02 (C-6), 103.92 (C-10), $116.18\left(\mathrm{C}-3^{\prime}, 5^{\prime}\right), 123.47$ (C-1'), $130.38\left(\mathrm{C}-2^{\prime}, 6^{\prime}\right)$, 136.54 (C-3), 147.63 (C-2), 158.03 (C-9), 160.34 (C-4'), 162.14 (C-5), 165.22 (C-7), 176.71 (C-4).

Quercetin (4): $\mathrm{C}_{15} \mathrm{H}_{10} \mathrm{O}_{7}$, m.p. $>300{ }^{\circ} \mathrm{C}$. UV $\lambda_{\text {max }} \mathrm{nm} \mathrm{MeOH}$ : 259, $308 \mathrm{sh}, 376$. +FAB-MC m/z: $303[\mathrm{M}+\mathrm{H}]^{+} .{ }^{13} \mathrm{C}-\mathrm{NMR}$ (DMSO-d, $125 \mathrm{MHz})$ : 93.74 (C-8), 98.47 (C-6), 104.81 (C-10), 115.21 (C-2'), 115.97 (C-5'), 121.04 (C-6'), 122.49 (C-1'), 134.71 (C-3), 145.33 (C-3'), 146.22 (C-2), 147.27 (C-4'), 158.74 (C-9), 161.52 (C-5), 164.67 (C-7), 178.53 (C-4).

$\beta$-Sitosterol-3-O-glucoside (5): $\mathrm{C}_{35} \mathrm{H}_{61} \mathrm{O}_{6}$, m.p. $281{ }^{\circ} \mathrm{C}$. $+\mathrm{FAB}-\mathrm{MC} m / z: 577[\mathrm{M}+\mathrm{H}]]^{+} .{ }^{13} \mathrm{C}-\mathrm{NMR}\left(\mathrm{CDCl}_{3}, 125\right.$ MHz): 11.62 (C-29), 12.46 (C-18), 18.35 (C-26), 19.37 (C-19), 19.83 (C-21), 20.51 (C-27), 21.82 (C-11), 23.70 (C-28), 25.72 (C-15), 25.94 (C-25), 29.48 (C-23), 29.74 (C-16), 29.93 (C-2), 33.07 (C-8), 33.19 (C-7), 37.15 (C-10), 37.38 (C-20), 38.82 (C-1), 39.73 (C-22), 40.33 (C-12), 42.97 (C-13), 44.01 (C-4), 50.21 (C-9), 50.41 (C-24), 56.26 (C-17), 57.31 (C-14), 61.58 (Glc, C-6'), 70.93 (Glc, C-4'), 74.77 (Glc, C-2'), 76.63 (Glc, C-5'), 77.02 (Glc, C-3'), 81.12 (C-3), 103.57 (Glc, C-1'), 122.15 (C-6), 140.52 (C-5).

Caffeic acid (6): $\mathrm{C}_{9} \mathrm{H}_{8} \mathrm{O}_{4}$, m.p. $224^{\circ} \mathrm{C}$. UV $\lambda_{\text {max }}$ nm MeOH: 238, $301 \mathrm{sh}, 325$. +FAB-MC m/z: $181[\mathrm{M}+\mathrm{H}]^{+} .{ }^{13} \mathrm{C}-\mathrm{NMR}$ (CD 3 OD, $125 \mathrm{MHz}): 114.92$ (C-8), 115.09 (C-2), 116.11 (C-5), 121.24 (C-6), 125.71 (C-1), 144.37 (C-7), 145.37 (C-3), 148.38 (C-4), 168.98 (C-9).

Gallic acid (7): $\mathrm{C}_{7} \mathrm{H}_{6} \mathrm{O}_{5}$, m.p. $250{ }^{\circ} \mathrm{C}$. UV $\lambda_{\max } \mathrm{nm} \mathrm{MeOH}$ : 275. +FAB-MC m/z: $171[\mathrm{M}+\mathrm{H}]]^{+} .{ }^{13} \mathrm{C}-\mathrm{NMR}$ (DMSO-d, $125 \mathrm{MHz}$ ): 109.08 (C-2, C-6), 120.47 (C-1), 137.84 (C-4), 145.11 (C-3, C-5), 167.74 (C-7).

3,5-Di-O-caffeoylquinic acid (8): $\mathrm{C}_{25} \mathrm{H}_{24} \mathrm{O}_{12}$, m.p. $170{ }^{\circ} \mathrm{C}$. UV $\lambda_{\max }$ nm MeOH: 220, 246 sh, 297 sh, 333. +FAB-MC $m / z: 517[\mathrm{M}+\mathrm{H}]^{+} .{ }^{13} \mathrm{C}-\mathrm{NMR}\left(\mathrm{CD}_{3} \mathrm{OD}, 125 \mathrm{MHz}\right): 74.62$ (C-1), 36.08 (C-2), 72.21 (C-3), 70.54 (C-4), 72.04 (C-5), 37.42 (C-6), $114.98\left(\mathrm{C}-8^{\prime \prime}\right), 115.20\left(\mathrm{C}-2^{\prime \prime}\right), 115.27$ (C-2'), $115.51\left(\mathrm{C}-8^{\prime}\right), 116.41\left(\mathrm{C}-5^{\prime \prime}\right), 116.41\left(\mathrm{C}-5^{\prime}\right), 123.01$ (C-6'), 123.42 (C-6"), 127.71 (C-1"), 127.81 (C-1'), 146.29 $\left(\mathrm{C}-3^{\prime \prime}\right), 146.74\left(\mathrm{C}-3^{\prime}\right), 147.08\left(\mathrm{C}-7^{\prime}\right), 147.37$ (C-7") 149.20 (C-4'), 149.37 (C-4"), 168.64 (C-9'), 168.91 (C-9'), 176.87 (C-7);

Isorhamnetin-3-O-glucoside (9): $\mathrm{C}_{15} \mathrm{H}_{10} \mathrm{O}_{6}$, m.p. $267{ }^{\circ} \mathrm{C}$. UV $\lambda_{\text {max }}$ nm MeOH: 258, 305, 361. +FAB-MC m/z: 479 $[\mathrm{M}+\mathrm{H}]]^{+} .{ }^{13} \mathrm{C}-\mathrm{NMR}\left(\mathrm{CD}_{3} \mathrm{OD}, 125 \mathrm{MHz}\right): 55.24\left(3^{\prime}-\mathrm{OCH}_{3}\right)$,
61.37 (Glc, C-6"), 71.48 (Glc, C-4"), 75.63 (Glc, C-2"), 76.40 (Glc, C-5"), 77.11 (Glc, C-3"), 94.37 (C-8), 99.12 (C-6), 104.52 (Glc, C-1"), 105.74 (C-10), 112.87 (C-2'), $114.12\left(\mathrm{C}-5^{\prime}\right), 123.24\left(\mathrm{C}-6^{\prime}\right), 123.97\left(\mathrm{C}-1^{\prime}\right), 135.22(\mathrm{C}-3)$, 148.61 (C-4'), 150.24 (C-3'), 158.14 (C-2), 158.61 (C-9), 164.27 (C-5), 166.12 (C-7), 179.21 (C-4).

Isoquercitrin (10): $\mathrm{C}_{21} \mathrm{H}_{20} \mathrm{O}_{12}$, m.p. $226{ }^{\circ} \mathrm{C}$. UV $\lambda_{\text {max }} \mathrm{nm}$ MeOH: 257, 359. +FAB-MC m/z: $465[\mathrm{M}+\mathrm{H}]^{+} .{ }^{13} \mathrm{C}-\mathrm{NMR}$ (CD $\mathrm{OD}, 125 \mathrm{MHz}$ ): 61.42 (Glc, C-6"'), 70.95 (Glc, C-4"), 75.31 (Glc, C-2"), 76.37 (Glc, C-5"), 77.25 (Glc, C-3"), 94.52 (C-8), 99.03 (C-6), 102.67 (Glc, C-1"), 103.04 (C-10), 114.93 (C-2'), 115.98 (C-5'), 122.54 (C-6'), 123.24 (C-1'), 135.74 (C-3), 144.35 (C-3'), 149.60 (C-4'), 159.05 (C-9), 160.71 (C-2), 163.58 (C-5), 165.81 (C-7), 179.55 (C-4).

3-O-Caffeoylquinic acid (11): $\mathrm{C}_{16} \mathrm{H}_{18} \mathrm{O}_{9}$, m.p. $206^{\circ} \mathrm{C}$. UV $\lambda_{\max }$ nm MeOH: 240, $302 \mathrm{sh}, 324$. +FAB-MC m/z: 354 $[\mathrm{M}+\mathrm{H}]^{+} .{ }^{13} \mathrm{C}-\mathrm{NMR}$ (CD $\left.\mathrm{OD}, 125 \mathrm{MHz}\right): 40.84$ (C-6), 38.72 (C-2), 71.54 (C-5), 72.67 (C-4), 73.27 (C-3), 77.41 (C-1), 115.14 (C-2'), 115.61 (C-8'), 116.51 (C-5'), 122.71 (C-6'), $127.84\left(\mathrm{C}-1^{\prime}\right), 146.50$ (C-3'), 146.90 (C-7'), 149.33 (C-4'), 169.51 (C-9'), 182.38 (C-7).

5-O-Caffeoylquinic acid (12): $\mathrm{C}_{16} \mathrm{H}_{18} \mathrm{O}_{9}$, m.p. $210^{\circ} \mathrm{C}$. UV $\lambda_{\text {max }}$ nm MeOH: 241, 305 sh, 326. +FAB-MC m/z: 354 $[\mathrm{M}+\mathrm{H}]^{+} .{ }^{13} \mathrm{C}-\mathrm{NMR}\left(\mathrm{CD}_{3} \mathrm{OD}, 125 \mathrm{MHz}\right): 39.18$ (C-2), 40.73 (C-6), 71.87 (C-3), 72.41 (C-4), 73.68 (C-5), 77.75 (C-1), 115.18 (C-2'), 115.52 (C-8'), 116.58 (C-5'), 122.93 (C-6'), $127.78\left(\mathrm{C}-1^{\prime}\right), 146.80\left(\mathrm{C}-3^{\prime}\right), 146.94\left(\mathrm{C}-7^{\prime}\right), 149.75$ (C-4'), 169.37 (C-9'), 181.10 (C-7).

Rutin (13): $\mathrm{C}_{27} \mathrm{H}_{30} \mathrm{O}_{16}$, m.p. $243{ }^{\circ} \mathrm{C}$. UV $\lambda_{\text {ma }} \mathrm{x}$ nm MeOH: 263, 364. +FAB-MC m/z: $611[\mathrm{M}+\mathrm{H}]]^{+} .{ }^{13} \mathrm{C}-\mathrm{NMR}$ (CD $\mathrm{OD}$, $125 \mathrm{MHz}$ ): 17.81 (Rha, C-6"' $), 68.31$ (Glc, C-6"'), 69.84 (Rha, C-5"'), 71.34 (Rha, C-2"'), 72.23 (Rha, C-3"'), 73.83 (Glc, C-4"), 74.34 (Rha, C-4"'), 75.68 (Glc, C-2"'), 77.25 (Glc, C-5"), 78.14 (Glc, C-3"), 94.63 (C-8), 99.82 (C-6), 102.08 (Rha, C-1"'), 104.51 (Glc, C-1"), 105.67 (C-10), 115.47 (C-2'), 116.67 (C-5'), 123.27 (C-6'), 123.75 (C-1'), 135.77 (C-3), 145.61 (C-3'), 149.92 (C-4'), 159.05 (C-9), 159.21 (C-2), 163.41 (C-5), 165.97 (C-7), 179.32 (C-4).

Narcissin (14): $\mathrm{C}_{28} \mathrm{H}_{32} \mathrm{O}_{16}$, m.p. $187{ }^{\circ} \mathrm{C}$. UV $\lambda_{\max } \mathrm{nm}$ MeOH: 254, 356. +FAB-MC m/z: $625[\mathrm{M}+\mathrm{H}]+{ }^{+}{ }^{13} \mathrm{C}-\mathrm{NMR}$ ( $\left.\mathrm{CD}_{3} \mathrm{OD}, 125 \mathrm{MHz}\right): 17.73$ (Rha, C-6"'), $55.01\left(3^{\prime}-\mathrm{OCH}_{3}\right)$, 68.37 (Glc, C-6"'), 69.52 (Rha, C-5"'), 71.18 (Rha, C-2'"'), 71.48 (Glc, C-4"), 71.82 (Rha, C-3"'), 74.59 (Rha, C-4"'), 75.63 (Glc, C-2"), 76.40 (Glc, C-5"), 77.11 (Glc, C-3"), 94.32 (C-8), 99.30 (C-6), 101.92 (Rha, C-1"'), 104.52 (Glc, C-1'), 105.11 (C-10), 112.96 (C-2'), 114.82 (C-5'), $123.02\left(\mathrm{C}-6^{\prime}\right), 123.74\left(\mathrm{C}-1^{\prime}\right), 135.18(\mathrm{C}-3), 148.71\left(\mathrm{C}-4^{\prime}\right)$, 150.02 (C-3'), 158.30 (C-9), 158.37 (C-2), 163.37 (C-5), 165.42 (C-7), 178.84 (C-4). 
Nicotiflorin (15): $\mathrm{C}_{27} \mathrm{H}_{30} \mathrm{O}_{15}$, m.p. $185{ }^{\circ} \mathrm{C} . \mathrm{UV} \lambda_{\max } \mathrm{nm}$ MeOH: 268, 351. +FAB-MC m/z: $595[\mathrm{M}+\mathrm{H}]^{+} .{ }^{13} \mathrm{C}-\mathrm{NMR}$ (CD OD, $125 \mathrm{MHz}$ ): 17.68 (Rha, C-6"'), 68.45 (Glc, C-6"'), 69.63 (Rha, C-5"'), 71.52 (Glc, C-4"'), 71.95 (Rha, C-2'"'), 72.27 (Rha, C-3"'), 74.45 (Rha, C-4"'), 75.71 (Glc, C-2"'), 76.94 (Glc, C-5"), 78.39 (Glc, C-3"), 94.75 (C-8), 99.97 (C-6), 102.18 (Rha, C-1"'), 104.49 (Glc, C-1"'), 105.71 (C-10), $116.40\left(\mathrm{C}-3^{\prime}, 5^{\prime}\right), 123.11\left(\mathrm{C}-1^{\prime}\right), 132.37$ (C-2',6'), 135.65 (C-3), 158.57 (C-9), 158.63 (C-2), 159.71 (C-4'), 161.82 (C-5), 166.07 (C-7), 179.29 (C-4).

Sucrose (16): $\mathrm{C}_{12} \mathrm{H}_{22} \mathrm{O}_{11}$, m.p. $185^{\circ} \mathrm{C}$. $[\alpha]_{\mathrm{D}}+65.9^{\circ}\left(\mathrm{H}_{2} \mathrm{O} ; \mathrm{c}\right.$ 5.0). ${ }^{13} \mathrm{C}-\mathrm{NMR}\left(\mathrm{D}_{2} \mathrm{O}, 125 \mathrm{MHz}\right): 62.82$ (Glc, C-6), 64.02 (Frc, C-1'), 65.05 (Frc, C-6'), 72.01 (Glc, C-4), 73.79 (Glc, C-2), 75.11 (Glc, C-5), 75.37 (Glc, C-3), 76.89 (Frc, C-4'), 79.18 (Frc, C-3'), 84.01 (Frc, C-5'), 95.15 (Glc, C-1), $106.21($ Frc, C-2').

\section{HPTLC-analysis conditions}

Powdered plant material $(500 \mathrm{mg})$ was extracted for $30 \mathrm{~min}$ with $25 \mathrm{~mL}$ of $\mathrm{MeOH}$ at $80^{\circ} \mathrm{C}$ in a water bath two times. The samples were then filtered through a cellulose acetate filter $(0.50 \mu \mathrm{m}$; Reakhim $)$ and concentrated. The residue was re-dissolved in $5 \mathrm{~mL}$ of $\mathrm{MeOH}$. The sample solutions were stored at $4{ }^{\circ} \mathrm{C}$ until used. Chromatography was performed on $15 \mathrm{~cm}$ x $10 \mathrm{~cm}$ Sorbfil-A silica gel HPTLC plates (layer thickness $0.1 \mathrm{~mm}$; Imid Ltd), which used without pretreatment. Standard $(1 \mu \mathrm{L})$ and sample solutions $(2 \mu \mathrm{L})$ were applied as $8 \mathrm{~mm}$ bands, $5 \mathrm{~mm}$ apart, $15 \mathrm{~mm}$ from the left of the plate and $15 \mathrm{~mm}$ from the bottom edge, by means of a APA-1 automatic sample applicator
(Imid Ltd). Plates were developed at room temperature $\left(20{ }^{\circ} \mathrm{C}\right)$ with toluene-EtOAc-HCOOH 5:4:1 (v/v, for flavonol aglycones, caffeic and gallic acids) or EtOAc1,2-dichloroethane-AcOH-HCOOH- $\mathrm{H}_{2} \mathrm{O}$ 10:2.5:1:1:0.8 (v/v, for flavonol glycosides and caffeoylquinic acids) as mobile phases, in a flat-bottomed vertical chamber previously saturated with mobile phase vapor (40 min); the development distance was $6 \mathrm{~cm}$. After development, plates were dried for $10 \mathrm{~min}$ in cool air and derivatized with Neu reagent (freshly prepared 0.5\% 2-aminoethyl diphenylborinate solution in EtOAc/5\% PEG 400 in 1,2-dichloroethane). After derivatization the plates were heated at $100{ }^{\circ} \mathrm{C}$ for 5 min with a TLC Plate Heater USP$1 \mathrm{M}$ (Imid Ltd) and then scanned $387 \mathrm{~nm}$ by use of a Sorbfil Videodensitometer 2.0 TLC Scanner (Imid Ltd) controlled by Sorbfil software; the slit dimensions were $3 \mathrm{~mm} \times 0.40$ $\mathrm{mm}$.

\section{Results and Discussion}

The 70\% EtOH-extract of Caragana bungei Ledeb., Fabaceae, flowering shoots was partitioned with $\mathrm{CHCl}_{3}$, EtOAc, and $n$-BuOH to yield three fractions which were separated by chromatographic column (gel permeation, silica gel and polyamide chromatography) and pTLC, given sixteen compounds. The isolated compounds including seven flavonoids [kaempferol (3), quercetin (4) (Olennikov et al., 2011b), isorhamnetin3-O-glucoside (9) (Manguro et al., 2004), isoquercitrin (quercetin-3-O-glucoside, 10) (Liu et al., 2010), rutin (quercetin-3-O-rutinoside, 13) (Hou et al., 2005), narcissin (isorhamnetin-3-O-rutinoside, 14) ( $\mathrm{Lu}$ et al.,

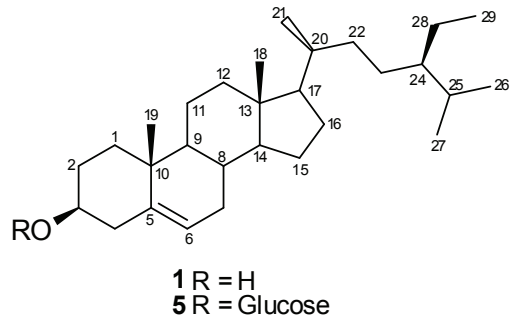<smiles>O=C(O)/C=C/c1ccc(O)c(O)c1</smiles>

6<smiles>O=c1ccc2ccc(O)cc2o1</smiles><smiles>O=C(O)c1cc(O)c(O)c(O)c1</smiles>

7<smiles>[R2]c1cc(-c2oc3cc(O)cc(O)c3c(=O)c2[R2])ccc1O</smiles>

$3 \mathrm{R}_{1} ; \mathrm{R}_{2}=\mathrm{H}$

$4 \mathrm{R}_{1}=\mathrm{H} ; \mathrm{R}_{2}=\mathrm{OH}$

$9 \mathrm{R}_{1}=$ Glucose; $\mathrm{R}_{2}=\mathrm{OCH}_{3}$ $10 \mathrm{R}_{1}=$ Glucose; $\mathrm{R}_{2}=\mathrm{OH}$ $13 \mathrm{R}_{1}=$ Rutinose; $\mathrm{R}_{2}=\mathrm{OH}$ $14 \mathrm{R}_{1}=$ Rutinose; $\mathrm{R}_{2}=\mathrm{OCH}_{3}$ $15 \mathrm{R}_{1}=$ Rutinose; $\mathrm{R}_{2}=\mathrm{H}$

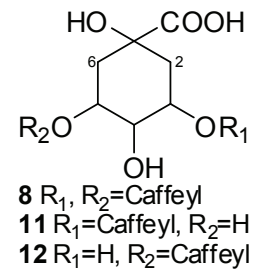<smiles>OC[C@H]1O[C@](O[C@@H]2O[C@H](CO)[C@H](O)[C@@H](O)[C@H]2O)([AlH2]O)[C@H](O)[C@@H]1O</smiles>

16 
Table 1. Phenolic compounds distribution, total phenolic content (TPC), total flavonoid content (TFC) and $\beta$-sitosterol content in $C$. bungei leaves, flowers and stems.

\begin{tabular}{|c|c|c|c|c|}
\hline Compound & Leaves & Flowers & Outer bark of stems & Inner bark of stems \\
\hline quercetin & $\operatorname{tr}^{\mathrm{a}}$ & tr. & + & tr. \\
\hline kaempferol & tr. & - & + & - \\
\hline isorhamnetin-3-O-glucoside & $+^{\mathrm{b}}$ & + & - & - \\
\hline isoquercitrin & + & + & - & - \\
\hline rutin & + & + & tr. & - \\
\hline narcissin & + & + & tr. & - \\
\hline nicotiflorin & + & - & - & - \\
\hline caffeic acid & + & - & - & - \\
\hline 3-O-caffeoylquinic acid & + & + & - & - \\
\hline 5-O-caffeoylquinic acid & + & + & - & - \\
\hline 3,5-di-O-caffeoylquinic acid & + & - & - & - \\
\hline gallic acid & tr. & - & + & - \\
\hline $\mathrm{TPC}, \mathrm{mg} / \mathrm{g}^{\mathrm{d}}$ & $40.09 \pm 0.82$ & $36.24 \pm 0.64$ & $12.60 \pm 0.38$ & $1.94 \pm 0.06$ \\
\hline $\mathrm{TFC}, \mathrm{mg} / \mathrm{g}^{\mathrm{d}}$ & $29.32 \pm 0.58$ & $18.67 \pm 0.37$ & $2.13 \pm 0.04$ & tr. \\
\hline$\beta$-sitosterol, mg/g $\mathrm{g}^{\mathrm{d}}$ & $1.39 \pm 0.04$ & $4.52 \pm 0.14$ & $1.43 \pm 0.04$ & $0.97 \pm 0.02$ \\
\hline
\end{tabular}

${ }^{\mathrm{a}}$ traces; ${ }^{\mathrm{b}}$ present; ${ }^{\mathrm{c}}$ not detected; ${ }^{\mathrm{d}}$ from dry plant weight.

2000), nicotiflorin (kaempferol-3-O-rutinoside, 15) (Hou et al., 2005)], four phenylpropanoids [caffeic acid (6) (Olennikov et al., 2011a), 3,5-di- $O$-caffeoylquinic acid (8) (Satake et al., 2007), 3-O-caffeoylquinic acid (11), 5-Ocaffeoylquinic acid (12) (Olennikov et al., 2011a)], two triterpenes $[\beta$-sitosterol (1), $\beta$-sitosterol-3- $O$-glucoside (5) (Tomimatsu \& Hashimoto, 1968)], umbelliferone (2) (Olennikov \& Tankhaeva, 2005a), gallic acid (7) (Olennikov et al., 2005b) and sucrose (16) (Jung et al., 2000) were identified by comparison of their m.p., $[\alpha]$ $\mathrm{D}, \mathrm{UV}, \mathrm{MS}$ and ${ }^{13} \mathrm{C}-\mathrm{NMR}$ data with those reported in the literature.

Accordingly to literature data some of isolated compounds were previously detected in other Caragana species: kaempferol in C. spinosa (Shpekina, 1990); quercetin in $C$. arborescens (Boinik et al., 1986), $C$. alaica (Butayarov et al., 1999), C. jubata (Umarov et al., 1971), C. pygmaea (Polovinko \& Yakovlev, 1985), C. sinica (Tai et al., 2010) and C. spinosa (Shpekina, 1990); isorhamnetin-3-O-glucoside in $C$. alaica (Butayarov, 1999); isoquercitrin in C. arborescens (Boinik, 1986), C. alaica (Butayarov, 1999), C. sinica (Tai et al, 2010) and $C$. spinosa (Shpekina, 1990); rutin in C. arborescens (Boinik, 1986), C. aurantiaca (Polovinko \& Shostakovskaya, 1987), C. pygmaea (Polovinko \& Yakovlev, 1985) and C. spinosa (Shpekina, 1990); narcissin in C. aurantiaca (Polovinko \& Shostakovskaya, 1987), C. pygmaea (Polovinko \& Yakovlev, 1985), C. sinica (Tai et al, 2010) and C. spinosa (Shpekina, 1990); $\beta$-sitosterol in $C$. conferta (Khan et al., 2009) and $C$. microphylla (Huo et al., 2007); $\beta$-sitosterol-3-O-glucoside in C. conferta (Khan et al., 2009); umbelliferone in C. frutex (Boinik et al., 1983); sucrose in C. microphylla (Huo et al., 2007). The presence of nicotiflorin, caffeic, 3-O-caffeoylquinic, 5-Ocaffeoylquinic, 3,5-di- $O$-caffeoylquinic and gallic acids was firstly shown for Caragana species in this work.

Using HPTLC analysis we were investigated the distribution of phenolic compounds in different $C$. bungei organs (leaves, flowers, stems). It was shown that leaves were concentrated the flavonoid glucosides and phenylpropanoids and traces of flavonoid aglycones and gallic acid (Table 1). The composition of flowers phenols was similar to those of leaves excluding of kaempferol, nicotiflorin, caffeic, 3,5-di-O-caffeoylquinic and gallic acids. Characteristic feature of stems was ability of outer bark (epidermis) to concentration of quercetin, kaempferol and gallic acid with absence or traces amounts of flavonoids and phenylpropanoids. Only traces of quercetin were detected in inner bark of stems. The values of total phenolic content in leaves, flowers, outer and inner barks of stems were $40.09,36.24,12.60$ and $1.94 \mathrm{mg} / \mathrm{g}$ from dry plant weight respectively, and values of total flavonoid content in leaves, flowers and outer bark of stems were 29.32, 18.67 and $2.13 \mathrm{mg} / \mathrm{g}$ from dry plant weight respectively.

The known data about biological activity of plant sterols as a hypocholesterolemic and antiatherosclerotic substances (Lees et al., 1977) was initiated the quantitative analysis of $\beta$-sitosterol in $C$. bungei. It was established that $\beta$-sitosterol contents in leaves, flowers, outer and inner barks of stems were $1.39,4.52,1.43$ and $0.97 \mathrm{mg} / \mathrm{g}$ respectively (Table 1 ). High content of $\beta$-sitosterol was allowed to consider $C$. bungei flowers as a potential 
raw material for production of hypocholesterolemic preparations.

\section{Conclusions}

The phytochemical study of Caragana bungei Ledeb. flowering shoots using in traditional medicine of Tuva andBuryatia Republics(RussianFederation) indicated that this plant species contain flavonols (kaempferol, quercetin, isorhamnetin-3-O-glucoside, isoquercitrin, rutin, narcissin, nicotiflorin), phenylpropanoids (caffeic acid, caffeoylquinic acids), triterpenes ( $\beta$-sitosterol, $\beta$-sitosterol-3-O-glucoside), coumarins (umbelliferone), simple phenols (gallic acid) and carbohydrates (sucrose) and can be regarded as perspective for further pharmacological investigation.

\section{Acknowledgment}

The authors acknowledge the financial support provided by the Presidium of Siberian Division of Russian Academy of Science under Project VI.52.1.3.

\section{References}

Aseeva TA, Dashiev DB, Dashiev AD, Nikolaev SM, Surkova NA, Chekhirova GV, Yurina TA 2008. Tibetan medicine of Buryats. Novosibirsk, SO RAN, Russia (in Russian).

Boinik VV, Batyuk NV, Kovalev VN 1986. Flavonoids of Caragana arborescens. Chem Nat Compd 22: 351-351.

Boinik VV, Kovalev VN, Komissarenko NF, Dikhtyarev VI 1983. Coumarins of the epigeal part of Caragana frutex. Chem Nat Compd 19: 742-742.

Butayarov AV, Batirov EKh, Tadzhibaev MM, Yuldashev MP 1999. Flavonoids of Caragana alaica. Chem Nat Compd 35: 628-630.

Chirikova NK, Olennikov DN, Tankhaeva LM 2010. Quantitative determination of flavonoid content in the aerial part of Baical Scullcap (Scutellaria baicalensis Georgi). Russ J Bioorg Chem 36: 915-922.

Hou W, Lin R, Lee T, Huang Y, Hsu F, Lee M 2005. The phenolic constituents and free radical scavenging activities of Gynura formosana Kiamnra. J Sci Food Agr 85: 615621.

Huo Y, Guo C, Lu S, Zhang Q, Qin L 2007. Chemical research of Caragana microphylla seeds. Chem Nat Compd 43: 214-215.

Jung MH, Kim HS, Ahn S, Kim ST, Jin MH, YimYH, Kim YK, Ok JH 2000. Complete assignment of the ${ }^{1} \mathrm{H}$ and ${ }^{13} \mathrm{C}$ NMR spectra of a sucrose ester from Euphorbia lathyris L. J Korean Magn Res Soc 4: 125-132.

Khan R, Malik A, Adhikari A, Qadir MI, Choudhary MI 2009. Conferols A and B, new anti-inflammatory 4-hydroxyisoflavones from Caragana conferta. Chem Pharm Bull 54: 415-417.
Lees AM, Mok HYI, Lees RS, McCluskey MA, Grundy SM 1977. Plant sterols as cholesterol-lowering agents: clinical trials in patients with hypercholesterolemia and studies of sterol balance. Atherosclerosis 28: 325-338.

Liu H, Mou Y, Zhao J, Wang J, Zhou L, Wang M, Wang D, Han J, Yu Z, Yang F 2010. Flavonoids from Halostachys caspica and their antimicrobial and antioxidant activities. Molecules 15: 7933-7945.

Lu Y, Sun Y, Foo LY, McNaab WC, Molan AL 2000. Phenolic glycosides of foliage legume Onobrychis viciifolia. Phytochemistry 55: 67-75.

Manguro LOA, Ugi I, Lemen P 2004. Further flavonol glycosides of Embelia schimperi leaves. B Chem Soc Ethiopia 18: 51-57.

Meng Q, Niu X, Roubin RH, Hanrahan JR 2009. Ethnobotany, phytochemistry and pharmacology of the genus Caragana used in traditional Chinese medicine. $J$ Ethnopharmacol 124: 350-368.

Olennikov DN, Stolbikova AV, Tankhaeva LM, Petrov EV 2011a. Phenylpropanoids and polysaccharides of Plantago depressa and P. media. Chem Nat Comp 47: 165-169.

Olennikov DN, Tankhaeva LM 2005a. Biologically active substances from Cacalia hastata L. 5. Triperpenes and coumarines. Chem Nat Compd 41: 600-601.

Olennikov DN, Tankhaeva LM,Agafonova SV 2011b. Antioxidant components of Laetiporus sulphureus (Bull.: Fr.) Murr. fruit bodies. Appl Biochem Micro 47: 419-425.

Olennikov DN, Tankhaeva LM, Nikolaeva GG, Nikolaev SM 2005b. Biologically active substances from Cacalia hastata L. 4. Phenolic acids. Chem Nat Compd 41: 222223.

Polovinko AE, Shostakovskaya NG 1987. Flavonoids of Caragana aurantiaca. Chem Nat Compd 23: 375-375.

Polovinko AE, Yakovlev GP 1985. Flavonoids of Caragana pygmaea. Chem Nat Compd 21: 252-253.

Satake T, Kamiya K, An Y, Oishi T, Yamamoto J 2007. The antitrombotic active constituents from Centella asiatica. Biol Pharm Bull 30: 935-940.

Shetty K, Curtis O F, Levin R E, Witkowsky R, Ang W 1995. Prevention of vitrification associated with in vitro shoot culture of oregano (Origanum vulgare) by Pseudomonas spp. J Plant Physiol 147: 447-451.

Shpekina GA 1990. Flavonoids of Caragana spinosa. Chem Nat Compd 26: 95.

Starek M, Krzek J, Michnik S 2007. TLC-densitometric analysis of $\beta$-sitosterol in pumpkin seed oil. J Planar Chromat 20: 327-330.

Tai Z, Cai L, Dai L, Sun W, Zhe W, Yang Y, Cao Q, Ding Z 2010. Antioxidant activities of Caragana sinica flower extracts and their main chemical constituents. Molecules 15: 6722-6732.

Tomimatsu T, Hashimoto M 1968. Studies on the chemical components of Rutaceae plants. II. Components of the root of Poncirus trifeliata Rafinesque. Yakugaku Zasshi 88: 1357-1359. 
Umarov A, Batyuk VS, Khaletskii AM 1971. Flavonols of Caragana jubata. Chem Nat Compd 7: 499-500.

\section{*Correspondence}

Daniil N. Olennikov

Laboratory of Medical and Biological Research, Institute of General and Experimental Biology, Siberian Division, Russian Academy of Sciences

Sakh'yanovoy str., 6, Ulan-Ude, Russian Federation, 670047

oldaniil@rambler.ru

Tel.: +7902 1600627

Fax: +7 3012433430 